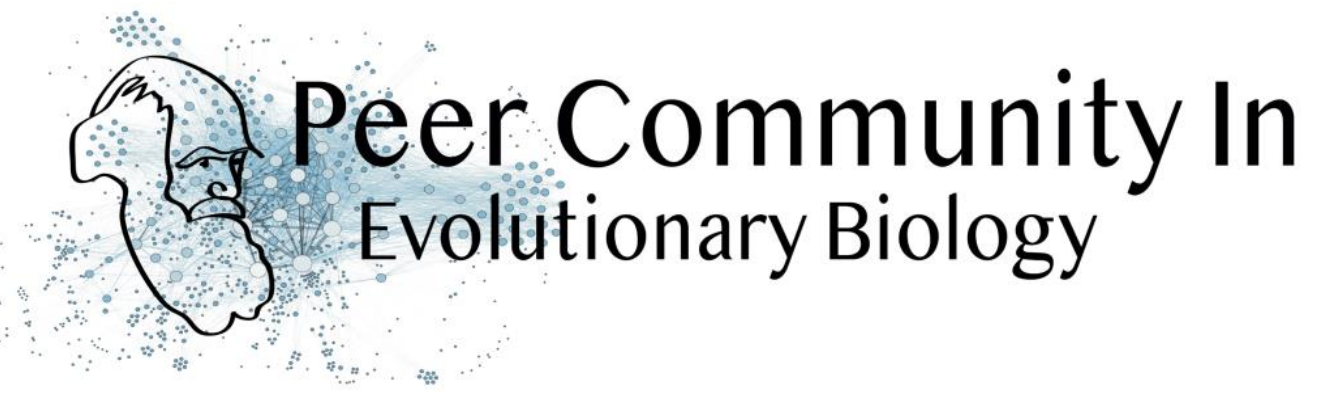

\title{
Nutrition-dependent effects of gut bacteria on growth plasticity in Drosophila melanogaster
}

Wolf Blanckenhorn based on reviews by Pedro Simões and 1 anonymous reviewer

\section{Open Access}

\section{A recommendation of:}

Robin Guilhot, Antoine Rombaut, Anne Xuéreb, Kate Howell, Simon Fellous. Environmental specificity in Drosophila-bacteria

Published: 20 November 2019

Copyright: This work is licensed under the Creative Commons Attribution-NoDerivatives 4.0 International License. To view a copy of this license, visit http://creativecommons.org/licen ses/by-nd/4.0/ symbiosis affects host developmental plasticity (2019), bioRxiv, 717702, ver. 3 peer-reviewed by Peer Community in Evolutionary Biology. 10.1101/717702

Submitted: 13 February 2019, Recommended: 20 November 2019 Cite this recommendation as:

Wolf Blanckenhorn (2019) Nutrition-dependent effects of gut bacteria on growth plasticity in Drosophila melanogaster. Peer Community in Evolutionary Biology, 100085.

10.24072/pci.evolbiol.100085

It is well known that the rearing environment has strong effects on life history and fitness traits of organisms. Microbes are part of every environment and as such likely contribute to such environmental effects. Gut bacteria are a special type of microbe that most animals harbor, and as such they are part of most animals' environment. Such microbial symbionts therefore likely contribute to local adaptation [1]. The main question underlying the laboratory study by Guilhot et al. [2] was: How much do 


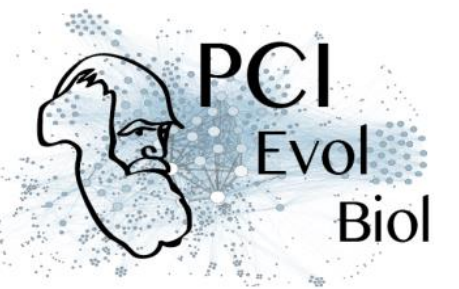

particular gut bacteria affect the organismal phenotype, in terms of life history and larval foraging traits, of the fruit fly Drosophila melanogaster, a common laboratory model species in biology? To investigate the above question, the authors isolated 4 taxa of bacteria from the gut of a (randomly picked) Drosophila melanogaster lab strain, and subsequently let Drosophila melanogaster eggs and larvae (stemming from their own, different lab strain) develop both in the typical artificial laboratory medium as well as in grapes, a natural "new" habitat for Drosophila larvae, inoculated with theses bacteria, singly and in combination, also including a bacteria-free control. By investigating various relevant developmental and size traits, the authors found that adding particularly Enterobacteria had some visible effects on several traits, both upward (indicting improvement) and downward (being detrimental) (with three other types of bacteria showing only minor or even no effects). In general, the grape medium reduced performance relative to the standard lab medium. Strongest interactive effects occurred for development time and body size, together making up growth plasticity [3], with lesser such effects on some related behavioral (feeding) traits (Figs. 2,3). The study premise is interesting, its general objectives are clearly laid out, and the practical work was conducted correctly as far as I can evaluate. The study remains largely descriptive in that no particular a priori hypotheses or predictions in relation to the specific bacteria isolated were formulated, not least because the bacteria were necessarily somewhat arbitrarily chosen and there were apparently no prior studies from which to derive concrete predictions. Overall, the results of this study should be of interest to the community of evolutionary ecologists, especially those working on nutritional and microbiome effects on animal life histories. I consider this work to be primarily ecological, with limited evolutionary content (e.g. no genetics) though some evolutionary implications, as mentioned in the paper's Conclusions. So this paper would best fit in a microbial or physiological ecology outlet/journal. The inclusion of a natural medium (grapes) must be commended because this permits inferences and conclusions for at least one natural environment, whereas inferences drawn from laboratory studies in the artificial medium that most Drosophila researchers seem to use are typically limited. Unsurprisingly perhaps, the study showed that Drosophila melanogaster fared generally better in the artificial than the chosen natural medium (grape). 


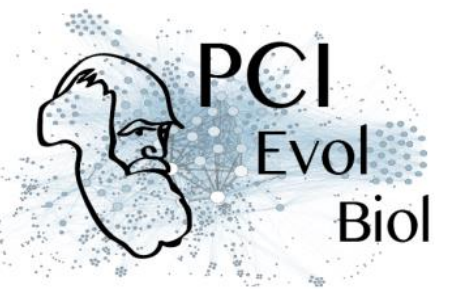

Crucially, however, the bacterial symbionts modified both media differentially. Although common bacterial taxa were chosen, the particular bacteria isolated and used remain arbitrary, as there are many. I note that the main and strongest interactive effects between medium and bacterial type are apparent for the Enterobacteria, and they probably also strongly, if not exclusively, mediate the overall effect of the bacterial mixture. While these specific data are novel, they are not very surprising. If we grow animals in different environments we can expect some detectable effects of these environments, including the bacterial (microbiome) environment, on the hosts life history. The standard and predicted [4] life history response of Drosophila melanogaster (but not all insects [3]) facing stressful nutritional environments, as apparently created by the Enterobacteria, is to extend development but come out smaller in the end. This is what happened here for the laboratory medium ([2]: Fig. 5). The biological interpretation is that individuals have more trouble ingesting and/or digesting the nutrients available (thus prolonging their foraging period and development), yet cannot convert the nutrients effectively into body size increments (hence emerging smaller). This is what the authors here refer to as developmental plasticity, which is ultimately nutritionally mediated. However, interestingly, a signal in the opposite direction was indicated for the bacterial mixture in the grape medium (flies emerging larger after accelerated development: Fig. 5), suggesting some positive effects on growth rate of the natural medium, perhaps related to grapes being a limited resource that needs to be escaped quickly [3]? The reversal of sexual size dimorphism across bacterial treatments in the grape environment detectable in Fig. 4 is interesting, too, though I don't understand why this happens, and this is not discussed. In general, more encompassing and increased questions in this context to be researched in the future could be: 1) are these effects predictable (not (yet) at this point, or so it seems); and 2) how strong are these environmental bacterial effects relative to other, more standard effects (e.g. relative to genetic variation, population variation, etc., or relative to other types of environmental effects like, say, temperature)? (3) It could further be asked why not natural but laboratory populations of Drosophila were used for this experiment, if the aim was to draw inferences for the wild situation. (4) Although Genotype $x$ Environment effects are invoked in the Discussion, they were not 


\section{Biol}

tested here, lacking genetically different Drosophila families or populations. From an evolutionary standpoint, I consider this the greatest weakness of the study. I was also not too thrilled by the particular statistical analyses employed, though this ultimately does not negate the results. Nevertheless, this work is a good start in this huge field investigating the microbiome. In conclusion, I can recommend this paper after review by $\mathrm{PCl}$ Evol Biol.

\section{References}

[1] Kawecki, T. J. and Ebert, D. (2004) Conceptual issues in local adaptation. Ecology Letters 7: 1225-1241. doi: 10.1111/j.1461-0248.2004.00684.x [2] Guilhot, R., Rombaut, A., Xuéreb, A., Howell, K. and Fellous, S. (2019). Environmental specificity in Drosophila-bacteria symbiosis affects host developmental plasticity. BioRxiv, 717702, v3 peer-reviewed and recommended by PCI Evolutionary Biology. doi: $10.1101 / 717702$

[3] Blanckenhorn, W.U. (1999) Different growth responses to temperature and resource limitation in three fly species with similar life histories. Evolutionary Ecology 13: 395-409. doi: 10.1023/A:1006741222586

[4] Stearns, S. C. and Koella, J. (1986) The evolution of phenotypic plasticity in life history traits: predictions of reaction norms for age and size at maturity. Evolution 40: 893-914. doi: 10.1111/j.1558-5646.1986.tb00560.x

\section{Revision round \#3}

\section{9-10-30}

- I might be missing something, but I don't question that (some of) the developmental plasticity you observe is mediated by microbes to some extent: indeed, in my view the mircobial composition affects the rearing environment, which in turn affects developmental plasticity.

- I still think the MANOVA analysis doesn't test what you want to test. In the ANCOVA I suggested you can test how microbe treatment/environment 


\section{Biol}

and food treatment affects the correlation between body size and development time by entering a three-way (or four-way) treatmentsizedevtime interaction.

- You can test for overall effects of symbiont treatment crossed with medium/grape food treatment all right. But within any treatment combination you cannot separate the (presumably random) effect of the food vs. the microbe environment, if this is what you are arguing. Again, I might be missing something.

- What remains is an acceptable descriptive paper on microbiome effects on developmental plasticity that is worth being published and will interest especially the community of scientists interested in the microbiome. I can give my OK for that.

Wolf Blanckenhorn

Preprint DOI: https://doi.org/10.1101/717702

\section{Author's reply:}

Thank you for your patience.

We posted a final version of our manuscript on bioRxiv. In this new work, we proceeded to two main changes.

First, we mentioned recent literature that focused on effects of microbial symbionts on host life history trade-offs. We think these results make more explicit the significance of our own findings.

Second, you spotted there were too many degrees of freedom in the analysis of age at emergence. Indeed, we had made a mistake in this model as we had forgotten to control for a form of pseudo-replication. We thus added to the model the relevant random term indicating the vial in which the flies had developed.

To facilitate your review of our changes, we attached a manuscript where modified text is in red. 


\section{Revision round \#2}

\section{9-08-29}

It is well known that the rearing environment has strong effects on life history and fitness traits of organisms. Microbes are part of every environment and as such likely contribute to such environmental effects. Gut bacteria are a special type of microbe that most animals harbor, and as such they are part of most animals' environment. How much do selected gut bacteria affect the organismal phenotype, in terms of life history and larval foraging traits, of the fruit fly Drosophila melanogaster, a common laboratory model species in biology? This was the main question underlying the laboratory study by Guilhot et al., which was previously assessed by two reviewers and myself for $\mathrm{PCl}$. To investigate the above question, the authors isolated 4 types/species of bacteria from the gut of their lab strain of Drosophila, and subsequently let Drosophila eggs and larvae develop in both the usual artificial laboratory medium as well as grapes (a natural habitat for Drosophila larvae) inoculated with theses bacteria, singly and in combination, plus a bacteria-free control. By investigating various relevant developmental traits, the authors found that adding particularly Enterobacteria had some visible improving effects on several traits (with three other types of bacteria showing only minor or even no effects), both upward (indicting improvement) and downward (being detrimental). In general, the grape medium reduced performance relative to the standard lab medium. Strongest interactive effects occurred for development time and body size, with lesser such effects on some related feeding behavioral traits (Figs. 2,3). The study overall was conducted correctly. In response to the previous reviews the Introduction was improved in the revised version to make clearer the general objectives of the study. The study remains largely descriptive in that no particular a priori hypotheses or predictions in relation to the particular bacteria isolated were tested, not least because the bacteria were somewhat arbitrarily chosen and there were apparently no particular prior studies from which to derive concrete predictions. Overall, the results of this study should be of interest to the 


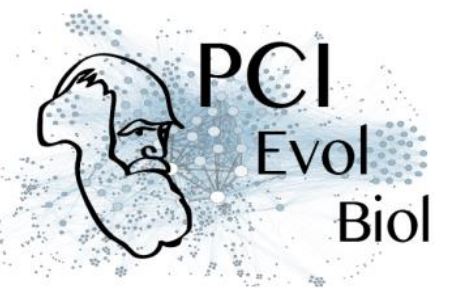

community of evolutionary ecologists, at least those working on nutritional and microbiome effects on animal life histories. As the strictly evolutionary content is limited (to non-existent), this paper would be best suited for a (microbial or behavioral or physiological) ecology outlet. The inclusion of a natural medium (grapes), in addition to the artificial lab medium, must be commended, because this should permit inferences and conclusions for at least one natural environment, as inferences drawn from laboratory studies for the natural situation are typically limited. The choice of bacteria isolated and used remains arbitrary, though there are many. Nevertheless a good start. It could be asked why not natural but artificial lab populations of Drosophila were used for this experiment, if the aim is to draw inferences for the wild situation. But again: a good start. While these specific data are novel, they are not very surprising. If we grow animals in different environments we can expect some detectable effects of these environments, including the bacterial (microbiome) environment, on the hosts life history. The standard and predicted (Stearns \& Koella 1986 Evolution) life history response of Drosophila melanogaster (but not all insects: Blanckenhorn 1999: Evol Ecol) facing stressful nutritional environments is to extend development but come out smaller in the end. This is what happened here (Figs. 3,4): size was smaller and development time longer in the grape medium, and some of the bacteria also weakly induced this (or the opposite) response. The biological interpretation is that individuals have more trouble ingesting and/or digesting the nutrients (thus prolonging their foraging period and development) and still cannot convert the nutrients effectively into body size increments (hence emerging smaller). This is what the authors here refer to as developmental plasticity, which is, still, ultimately nutritionally mediated. That is, the conclusion that this is not mediated by resource acquisition is misguided. I point out that the main and strongest interactive effects between medium and bacterial type are really only apparent for the enterobacteria, and they probably also mediate the overall effect of the mixture. For simplicity, I therefore suggest to initially present all data, but, in a second step, to only analyse control, enterobacteria \& mixture, which would make plots and analyses much simpler and clearer. I say this because the new analyses presented in response to our previous statistical comments are actually no improvement whatsoever, but worse than before. In 


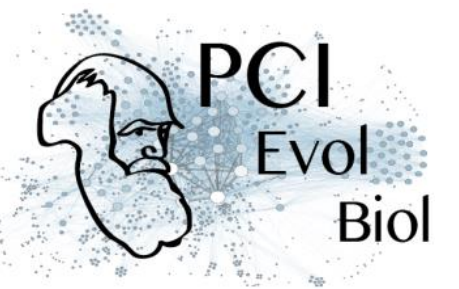

general, more encompassing and increased questions in this context to be researched in the future could be: 1) are these effects predictable (not (yet) at this point, or so it seems), and 2) how strong are these environmental bacterial effects relative to other, more standard effects (e.g. relative to genetic variation, population variation, etc., or relative to other types of environmental effects like, say, temperature)? Although Genotype x Environment effects are invoked, they were not tested here due to lacking genetically different Drosophila families or populations tested. I consider this a major weakness of the study because it does not allow comparisons with other environments or situations. So, while interesting in principle, the study appears a bit basic in the end. As mentioned before, and outlined above, the negative correlation between development time and body size is expected in stressful nutritional environments, which the enterobacteria apparently create. The multivariate analysis presented (which in the end analyses the effects of fruit salad instead of apples and oranges separately) is not what should be done; authors need to test for a correlation (between size and development time) within treatments properly with ANCOVA, entering ALL main factors (medium, bacteria, sex, plus interactions; cf. Table 1), such that the remaining correlation is indeed tested WITHIN treatment combinations. The negative regression ACROSS treatments is not really relevant because the tested environments are arbitrary. Suffice it to show that body size declines relative to control, while development time prolongs much more in relation (Fig. 4). That this somewhat differs between the two nutritional treatments is interesting, of course. I don't see much "accelerated" but mostly decelerated development, in standard medium females in particular. The flies grow better in standard medium than on grapes (Fig. 4), which is perhaps a bit surprising. The reversal of sexual size dimorphism across bacterial treatments in the grape environment visible in Fig. 3 is interesting too, though I don't understand why this happens. If I understand correctly your sample size should be 15 (replicates) $\times 2$ nutritional media $\times 6$ bacteria treatments $=180$. When analysing sexes separately this number doubles. The latter should be the magnitude of the error term in your full analyses. I don't see a need to analyse treatment means (you can plot them all right!); just present the overall ANOVAs of Table 1 (plus a corresponding ANCOVA; see above). (And reduce the data set to 
3 bacteria treatments to do the same analysis, as suggested above.) I found it difficult to edit the tracked-changes version, which did not save my comments after all. In summary, some further revisions are in order and then the paper could be recommended by $\mathrm{PCI}$ EvolBiol.

Wolf Blanckenhorn, University of Zurich

Preprint DOI: https://doi.org/10.1101/717702

\section{Author's reply:}

\section{Download author's reply (PDF file)}

\section{Revision round \#1}

\section{9-03-18}

It is well known that the rearing environment has strong effects on life history and fitness traits of organisms. Microbes are part of every environment and as such likely contribute to such environmental effects. Gut bacteria are a special type of microbe that most animals harbor, and as such they are part of the animal's environment. How much do selected gut bacteria affect the phenotype, in terms of life history and larval foraging traits, of the fruit fly Drosophila melanogaster, a common laboratory model species in biology? This was the main aim of the laboratory study by Guilhot et al. that was herewith assessed by two reviewers. To investigate the above question, the authors isolated 4 types/species of bacteria from the gut of their lab strain of Drosophila, and subsequently let Drosophila eggs and larvae develop in both the usual artificial laboratory medium and grapes (a natural habitat for Drosophila larvae) inoculated with theses bacteria, singly and in combination, plus a bacteria-free control. By investigating various relevant developmental traits, the authors found that adding the bacteria DID have some visible (significant) effects on various traits, both upward (indicting improvement) and downward (being detrimental). The study overall appears well conducted and presented. The text is largely clear, though the reviewers point out to some unclarities, which can and should be fixed. There are no major 


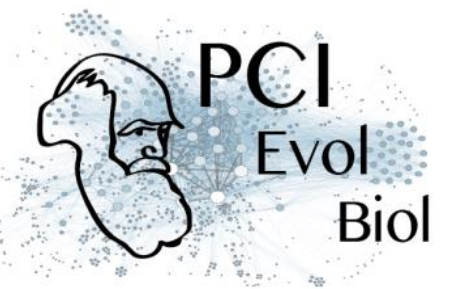

errors. As is, the study is largely descriptive, i.e. does not test particular hypotheses or predictions in relation to the particular bacteria isolated, not least because the bacteria were chosen ad (post) hoc and there were apparently no particular prior studies from which to derive concrete predictions. The inclusion of a natural medium (grapes), in addition to the artificial lab medium, must be commended, because this should permit inferences and conclusions for at least one natural environment, as inferences drawn from laboratory studies for the natural situation are typically limited. Nevertheless, it remains unclear why these, and not other bacteria were chosen, further limiting general inferences. It also remains unclear why not natural but artificial lab populations of Drosophila were used, if the aim is to draw inferences for the wild situation. But this is a good first start. While the data are novel, the fact that some detectable effects of the bacterial environment on life history traits is present is not really surprising, as the reviewers point out. More interesting questions would be: 1 ) are these effects predictable (not (yet) at this point, or so it seems), and 2) how strong are these environmental bacterial effects relative to other, more standard effects (e.g. relative to genetic variation, population variation (cf. reviewer 2), or relative to other types of environmental effects like, say, temperature)? Although Genotype $x$ Environment effects are invoked, they were not tested here due to lacking genetically different Drosophila families or populations tested. I consider this a major weakness of the study because it does not allow comparisons to other environments or situations. So, while interesting in principle, the study appears a bit arbitrary in the end. The reviewers point out a number of weaknesses and unclarities, to which I add my own. Many will be fixable, and should be fixed, in a revision. But to make this study more meaningful and interesting for the community it would be best to add some additional treatment levels as suggested above (different families, populations, perhaps another environmental factor, etc.). The questions addressed are not laid out clearly in the Introduction. What WERE the main questions/expectations/predictions? Why these and not other bacteria? The role of the "evolutionary" part regarding one bacterium class is not related to the rest of the study, which is not evolutionary at all but mainly physiological or developmental. Hence the title is a misnomer. The negative correlation between development time and body size needs to be tested properly 
with ANCOVA, entering ALL main factors (medium, bacteria, sex, plus interactions; cf. Table 1), such that the remaining correlation is indeed tested WITHIN treatment combinations. Fig. 3 clearly indicates that the negative regression line largely results from the two medium treatments (red vs. blue dots; lab vs. grape). I don't think this is what the authors are saying or testing. Overall, this descriptive study is worth publishing after major revision as indicated by the reviewers and myself above. Better still, adding further treatment levels would make this work more interesting and meaningful.

Additional requirements of the managing board: As indicated in the 'How does it work?' section and in the code of conduct, please make sure that: -Data are available to readers, either in the text or through an open data repository such as Zenodo (free), Dryad (to pay) or some other institutional repository. Data must be reusable, thus metadata or accompanying text must carefully describe the data. -Details on quantitative analyses (e.g., data treatment and statistical scripts in R, bioinformatic pipeline scripts, etc.) and details concerning simulations (scripts, codes) are available to readers in the text, as appendices, or through an open data repository, such as Zenodo, Dryad or some other institutional repository. The scripts or codes must be carefully described so that they can be reused. -Details on experimental procedures are available to readers in the text or as appendices.

-Authors have no financial conflict of interest relating to the article. The article must contain a "Conflict of interest disclosure" paragraph before the reference section containing this sentence: "The authors of this preprint declare that they have no financial conflict of interest with the content of this article." If appropriate, this disclosure may be completed by a sentence indicating that some of the authors are $\mathrm{PCl}$ recommenders: "XXX is one of the $\mathrm{PCl}$ Evol Biol recommenders."

Preprint DOI: https://doi.org/10.1101/546838

Reviewed by anonymous reviewer, 2019-02-18 12:04

The aim of this manuscript is to explore whether (and how) interactions between symbionts depend on the environment where they are tested. For that the authors isolated four bacterial strains from a Drosophila melanogaster laboratory 
population and tested how they affected several larval and adult traits when tested in two different media: (ancestral) laboratory media and grape berry media with live yeast. In particular, they recorded larval size, mouthpart movement speed, number of visible larvae, survival until emergence and a proxy for adult size. Additionally, they analyzed the microbial content of recently emerged adults and tested metabolic profiles of two of the bacterial isolates and of Actinobacteria that were present in grape berry medium at the end of the experiment. In general, the authors observed that the effect of the bacteria on the several traits assayed differed between the laboratory and grape fruit environment. In fact, in several traits some of the bacterial strains change from beneficial to deleterious indicating the dangers of generalizing from laboratory studies to the natural environment.

This study tackles the impact of environmental variation in the symbiont effect on the host and how it varies between different symbionts strains. This is both interesting from the point of view of understanding how symbionts may constraint or promote adaptation to new conditions, but it also raises an important point on whether observations done in laboratory settings can be taken as a proxy of what happens in natural conditions. However, I think this manuscript needs some revision, mainly the statistical methods, results section and the discussion. I divided my comments into major and minor comments (in no particular order) and hope that these suggestions may help to improve the manuscript.

Major comments I found it hard to understand what were the questions that you want to address with this study. In order to make it clearer I would suggest adding the main questions of the study at the end of the introduction (and some hypotheses for each). Then these main questions could be used in the results, material and methods and discussion section as titles to better guide the reader.

L205:206 - Why are you profiling these two bacteria in specific and not the other ones? What does the profile mean in terms of the impact of the bacteria on the host? Would it be worth to test whether some specific metabolic profiles have a specific impact on the host? This is something that I think it's missing in the manuscript to explain why you did the profiling. 
Data analysis - Instead of the post-hoc students test you should use a Tukey test, as it will correct for multiple comparisons and do the comparison considering the variance of the whole data set and not only the two levels you are comparing. An interesting analysis would be to perform a PCA using all traits analysed to see how different are the effects of bacterial strains and environments in a multivariate phenotype.

- While I see the interest of analyzing the Actinobacteria that seemed to evolve in the medium without flies, I do not think it makes sense in the context of this work (as you are studying symbiosis and how it varies across environments) but not any kind of evolution per se, so I would remove it from the manuscript. Moreover, I do not think you can take a lot of conclusions from a single replicate vial that evolved a particular kind of strain.

Discussion: Symbiont-mediated evolution section - I have a problem with this section, as you don't really test evolution of the Drosophila populations with the different treatments. I don't think you can actually say whether bacteria are helpful, neutral or detrimental for adaptation per se. You can just say whether they have a different effect on grape medium or on laboratory medium in a single generation. Moreover, without studying the evolution of the populations you cannot disentangle whether your results stem from other sources of variation such as maternal effects or plasticity. Thus, this section seems to be rather large and extended for the amount of evidence you provide.

- Figures 1, 2, - These figures are really hard to understand. I suggest that you remove the lines between the points (as the $x$ axis has categorical variables and the line plot are usually used for continuous variables, such as time or gradients). I think that bar plots would be easier to visualize instead of the scatter points. The letters are also really confusing. To add the information about significantly different comparisons you could use a matrix plot with all combinations of environments*Treatment in both axis and color code each comparison for the different levels of significance.

Minor comments L67:68 - It is not true that the most striking difference between laboratory and the wild is the substrate where the flies eat. There are a 


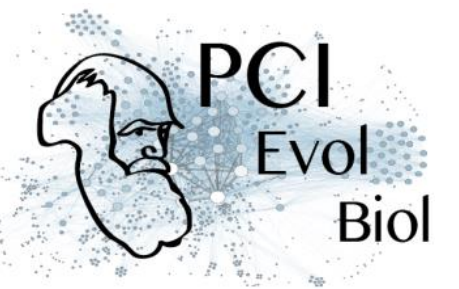

large number of other variables, such as population size, presence of competitors and predators, high variability in weather conditions, etc that also play a BIG role on how species live and reproduce. This sentence should be rephrased. L80 recorded sounds weird, maybe "scored" would be a better term L 143 - It would be easier/better for the reader if you had a supplementary figure explaining the experimental design. L 163:164 - The mixed and single bacterial isolates should have the same of bacterial density in the beginning. This way you may have an effect in the mixed treatment just because of the overall bacterial density. In fact, you observed that for the actinobacteria the inoculate size is actually important.

L184:186 - Maybe a simpler of saying this is to say that for each vial the sex of the flies to measure was chosen randomly. Statistics - In order to extract the mean value of the environment you should subtract it from the values, and not divide, as dividing will change the variance of the data, and that's what you are analyzing with your statistical analyses. L203:204 - I would remove this sentence altogether L255 - I think it's incorrect to say that the bacteria decreased directly the movement of the mouthparts, or any other phenotype. A more correct may would be to say that in the actinobacteria treatment there was a slowing down of the movement. You should correct this kind of use of the direct causality between bacteria and phenotype in other instances of the manuscript (e.g. L245, L247, etc) L 270 - was influenced L325 - Comparison of what? L339:341 - This phrase is very hard to read. Please rephrase into a simpler form L341 - There are many differences between the natural and laboratory environment. Thus, I suggest you should change ALL instances of close to closer. L360 - Why does the microbial growth on the surface of the vial without flies supports the hypothesis that these bacteria are eaten by the larvae? This could also happen if the larvae mix the medium while moving, and destroy the colonies and substrate, making the development of the white microbial growth impossible in their presence. L387:390 - Any clue why would this happen? Does grape have less nutrients than in laboratory medium and so the actinobacteria are an added stress? L395:397 Not really sure you can say this without a reference or showing data. So I would remove this part L451:452 - Grammatically this sentence is not correct. I think you should remove the "then". I am also unsure whether the sentence scientifically correct as the genetic ability to deal with stress of the host is not the 
only thing that defines bacteria to act as parasites or mutualists. For example, coevolution in the laboratory environment led Wolbachia to change from parasite to mutualist in D. simulans laboratory populations (Weeks et al 2007 Plos Biol.) L461 - "in the" instead of "on the" Conclusions - I would rephrase the last sentence with something simpler and to the point. Maybe something like "Our results demonstrate that in order to understand the ecology and evolution of symbiotic interactions in the wild it is necessary to use ecologically realistic conditions."

\section{Reviewed by Pedro Simões, 2019-02-18 12:04}

I think this is a nice study that addresses a very timely topic, the interactions between bacteria and hosts and their effects in different environments. The finding that the effects of bacteria on host are environment-specific is interesting (although not particularly surprising) and can lead to new research directly targeting the evolutionary implications of such interactions for the hosts (as well as bacteria). Additionally, it would be important to complement this study with the analysis of other populations to test if the observed effects are general or population-specific. My major concern regarding the study lies on the interpretation and discussion of the findings associated with 1) host developmental plasticity (specifically data from fig. 3) and 2) bacterial evolution (data from fig S6) - I detail these in the major comments below.

Major comments: 1) Lines 417-420 -The observed negative trend between larval development speed and adult size is expected due to a physiological trade-off, as faster development likely leads to lower adult size in Drosophila. In this case, bacteria that lead to an increase in larval development speed likely originate a correlated effect of lower adult size. Please explain better this part of the discussion. It might also help to clearly state the expectations for this analysis in the introduction (see comment below, lines 83-85). Overall, this data is a bit fragile as it is based on the combined data across environments (and there are no analyses testing for differences between environments in this relationship). 2) Lines 488-493 - I do not see evidence for the referred adaptation to grape. From Fig S6 I conclude that in general actinobacteria reduce in number with increasing 


\section{Biol}

time in grape. Please explain better your interpretation. Also there is no figure S6.1 3) Lines 472-502 - I would reduce this paragraph in size so to downplay its overall importance in the manuscript. I think it is a bit too speculative considering the strength of the data obtained. (see comment above about fig S6).

Specific Comments: Line 15 - replace "adaptation" by "conditions". Line 33 replace "participate" by "contribute". Line 34 - replace "to" by "for". Lines 8385 - expectations for this analysis should be clearly stated in the introduction. Lines 90-92 - some more info here would be helpful (e.g. number of founders, generations in the new laboratory). Lines 97-99 - is it possible to have an idea of the relative abundance of the bacteria chosen in the whole bacterial community of this D. melanogaster strain? Lines 223-224 - Was a correction for multiple testing applied? It might be adequate as several comparisons were made. Lines 266-267 - I would say "means not connected by at least one shared letter are significantly different." Lines 305-307 - was this tested statistically? if so please refer test statistics. It might be relevant to do so for both males and females, as in both cases heterogeneity is observable between environments. Line $333-1$ think you mean figure S5.2A. Line 335 - I think you mean figure S5.2B. Also fig S5.1 should be referred in this section. Lines 339-341 - I had trouble understanding this sentence, please rephase it. Also I would state here the major finding of the study: that the effects of different symbionts on host phenotype are environment-dependent. Lines 399-401 - I don't see how this conclusion can result from figure 4. Actually I don't think this can be concluded at all (based on the data from fig S6) - see also comments 2) and 3). Lines 411-413 - Remove or move to methods. Line 470 - replace "participate" by "contribute".

\section{Author's reply:}

\section{Download author's reply (PDF file)}

